\title{
Pitiriasis rubra pilaris juvenil. Presentación de un caso tratado con etanercept
}

\section{Juvenile pityriasis rubra pilaris. A case report treated with etanercept}

\author{
Víctor M. Gutiérrez-San Lucas, Isabel Talledo-Mera*, Ingrid de los Ángeles Masson-Pinto, \\ Johana M. Romo-Erazo y Carmen López-Acosta \\ Servicio de Dermatología, Hospital de Especialidades “Teodoro Maldonado Carbo”, Guayaquil, Ecuador
}

\section{Resumen}

La pitiriasis rubra pilaris es una dermatosis infrecuente cuya etiología se desconoce; se caracteriza por pápulas foliculares hiperqueratósicas, placas eritematosas y escamosas que pueden progresar a eritrodermia, con islas de piel sana, y queratodermia palmoplantar. Los subtipos III, IV y V, según la clasificación de Griffiths, se observan exclusivamente en niños. El tratamiento de esta dermatosis constituye un desafío: no existe un tratamiento universal aceptado ya que la tasa de respuesta a diferentes modalidades varía de manera amplia. Se presenta el caso de una pitiriasis rubra pilaris juvenil de tipo III con buena respuesta a etanercept.

Palabras clave: Pitiriasis rubra pilaris. Juvenil. Tratamiento. Etanercept.

\section{Abstract}

Pityriasis rubra pilaris is a rare skin disease whose etiology is unknown, characterized by hyperkeratotic follicular papules, scaly plaques that can progress to erythroderma, with islands of sparing, and palmoplantar keratoderma. Subtypes III, IV, and $V$ according to the classification of Griffiths, are observed exclusively in children. The treatment of this dermatosis constitutes a challenge, there is no universally accepted treatment since the response rate to different therapies varies widely. We present the case of a type III juvenile pityriasis rubra with a good response to etanercept.

Key words: Pityriasis rubra pilaris. Juvenile. Treatment. Etanercept.

\section{Introducción}

La pitiriasis rubra pilaris (PRP) es un trastorno de la queratinización poco frecuente y de causa desconocida. Griffiths propuso una clasificación, basada en la edad, duración y características de la afectación cutánea, en seis tipos: I, típica del adulto; II, atípica del adulto; III, típica juvenil; IV, circunscrita juvenil; V, atípica juvenil ${ }^{1}$, con la adición más adelante del tipo VI relacionado con HIV². Los tipos III, IV y V se presentan en edades pediátricas y representan el $40 \%$ de los pacientes con PRP, con una mayor afectación en los varones, en una proporción de 3:2 con respecto a las mujeres $^{3}$. La forma típica juvenil se caracteriza por
Correspondencia:

*Isabel Talledo-Mera

E-mail: talledoisabel89@gmail.com
Fecha de recepción: 18-07-2020 Fecha de aceptación: 25-10-2020 DOI: 10.24875/MCUT.20000103
Disponible en internet: 01-09-2021 Med Cutan Iber Lat Am. 2021;49(3):148-151 www.MedicinaCutanealLA.com 0210-5187/C 2020 Colegio Ibero Latinoamericano de Dermatología A.C. (CILAD). Publicado por Permanyer. Este es un artículo open access bajo la licencia CC BY-NC-ND (http://creativecommons.org/licenses/by-nc-nd/4.0/). 
eritrodermia con islotes de piel indemnes/sanos, pápulas foliculares hiperqueratósicas sobre una base eritematosa, de distribución generalizada, con o sin queratodermia palmoplantar difusa, de larga duración (>20 años). El tratamiento de esta dermatosis tanto en adultos como en niños es todavía un desafío, ya que no existe un consenso universal aceptado porque la tasa de respuesta a diferentes tratamientos varía de forma amplia. La mayor parte de los estudios presentados en edad pediátrica se ha tratado con compuestos tópicos solos o combinados, fototerapia y retinoides sistémicos. Los fármacos biológicos constituyen una opción terapéutica prometedora tanto en la PRP en adultos y la juvenil como en el tratamiento de tercera línea.

\section{Caso clínico}

Se presenta el caso de un adolescente masculino de 15 años de edad sin antecedentes patológicos personales ni familiares de relevancia. Se lo deriva al servicio por presentar dermatosis de un año de evolución con lesiones cutáneas localizadas inicialmente en la parte superior del tronco y que se extienden al abdomen, espalda y extremidades. Se diagnosticó antes con psoriasis y recibió tratamiento con corticoides tópicos, retinoides sistémicos y metrotexato $(15 \mathrm{mg} / \mathrm{sem})$ durante un año sin mejoría clínica evidente. La exploración física identificó pápulas foliculares hiperqueratósicas en miembros inferiores, acompañadas de placas eritematosas y descamativas con un tono rojo-anaranjado en tronco y extremidades, y en particular un intenso eritema con áreas indemnes (Fig. 1), junto con prurito y dolor a la palpación. No había afectación de palmas y plantas. No se reconoció compromiso de mucosas ni síntomas sistémicos. Con la sospecha clínica de PRP, se realizó biopsia de piel con tinción de hematoxilina-eosina, que reveló acantosis moderada psoriasiforme irregular con montículos de paraqueratosis en focos que alternaban con áreas ortoqueratósicas, espongiosis y exocitosis de linfocitos (Fig. 2); se confirmó el diagnóstico de pitiriasis rubra pilaris juvenil de tipo III. Se inició tratamiento con ciclosporina ( $3 \mathrm{mg} / \mathrm{kg}$ de peso) por cuatro semanas sin mejoría clínica y con posterioridad se inició etanercept (50 mg/sem), con remisión clínica después de cuatro semanas de tratamiento. Se observó una franca mejoría con disminución de eritrodermia y placas eritematosas y descamativas, y se logró un aclaramiento al cabo de dos meses (Fig. 3). No se identificaron efectos secundarios durante el curso del tratamiento. Hasta el

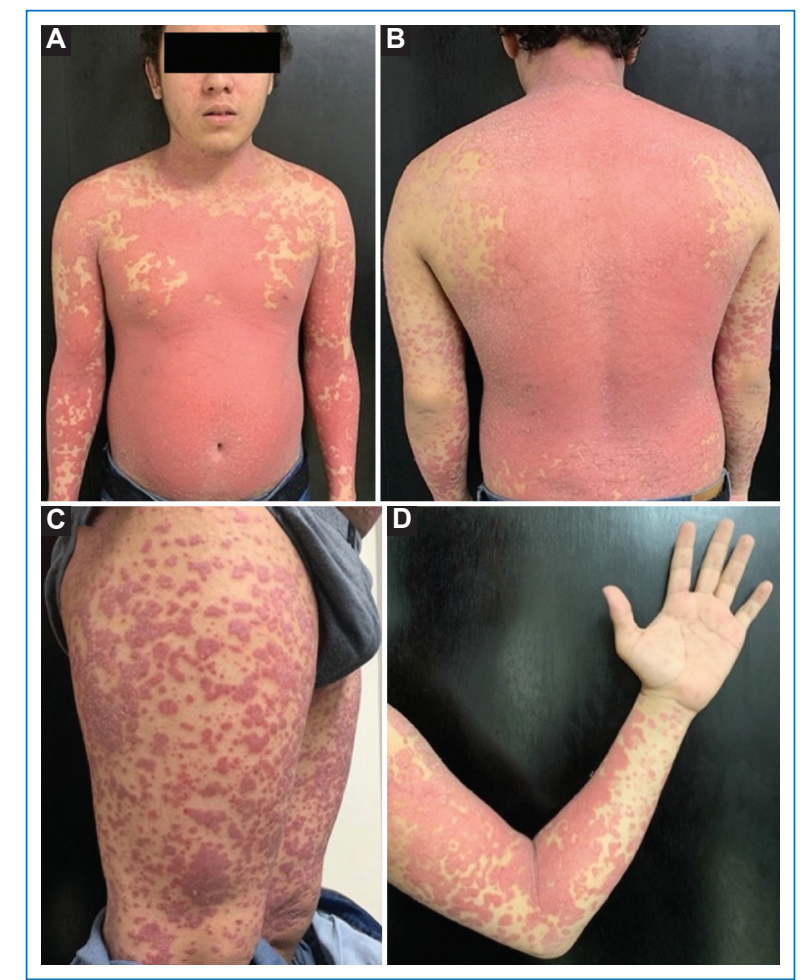

Figura 1. A-B: placas eritemato-descamativas con islotes de piel sana en tronco. C-D: pápulas foliculares hiperqueratósicas en extremidades.

momento, el paciente continúa con etanercept a dosis de $50 \mathrm{mg} / \mathrm{sem}$ (36 dosis recibidas hasta ahora).

\section{Discusión}

La PRP es un dermatosis eritematosa y descamativa, que describió en 1856 Devergie como un trastorno papulo-escamoso raro, que en 1889 Edouard Besnier denominó pitiriasis rubra pilaris 4 . Es una anomalía queratósica papulo-escamosa de etiología y patogenia desconocidas que se ha relacionado con el metabolismo anormal de la vitamina $A$, con una heterogenicidad clínica considerable. Se ha demostrado que esta entidad tiene tres grupos de presentación: primera infancia (0-10 años), infancia tardía (11-19 años) y adultez (40-60 años). Además, en la infancia, los hombres se ven afectados con mayor frecuencia que las mujeres (relación 3:2), a diferencia de la PRP del adulto en la que ambos sexos se ven afectados por igual ${ }^{3}$. Griffiths clasificó esta dermatosis en cinco subtipos, con base en la edad de inicio, la evolución, la apariencia clínica y el pronóstico, y de los cuales los tipos III (típica juvenil), IV (circunscrita juvenil) y V (atípica juvenil) se presentan exclusivamente en niños ${ }^{1}$; más 


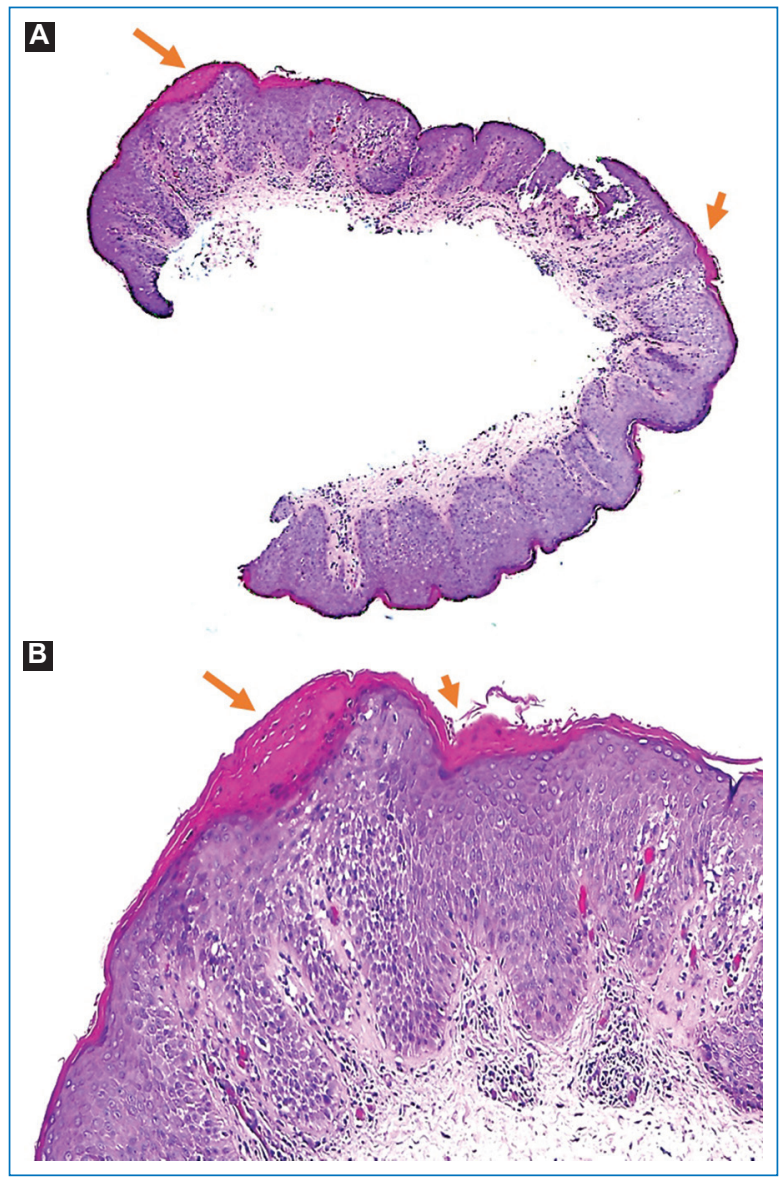

Figura 2. A: secciones histológicas de piel con acantosis moderada psoriasiforme irregular con montículos de paraqueratosis en focos (flechas), que alternan con áreas ortoqueratósicas $(H-E, 2.5 x)$. B: a mayor aumento se observa una zona de paraqueratosis con espongiosis y exocitosis de linfocitos a diferentes niveles (H-E, 10x).

adelante en 1995 se agregó el tipo VI relacionado con pacientes con $\mathrm{VIH}^{2}$.

El tipo III o típico juvenil afecta al $10 \%$ de los pacientes y es la forma más común en la edad pediátrica; se presenta por lo general entre los cinco y 10 años de edad y sus características clínicas son idénticas a las del PRP de tipo I o típica del adulto. Las más de las veces, las lesiones comienzan en la cabeza, el cuello o la parte superior del tronco, y a continuación se generalizan. De manera inicial se presentan máculas eritemato-escamosas que pronto desarrollan pápulas foliculares o perifoliculares, con un tapón queratósico acuminado central. Al final, estas pápulas se fusionan para formar placas eritemato-descamativas, que de modo característico se extienden en dirección cefalocaudal con un tono rojo-anaranjado; en ocasiones, la

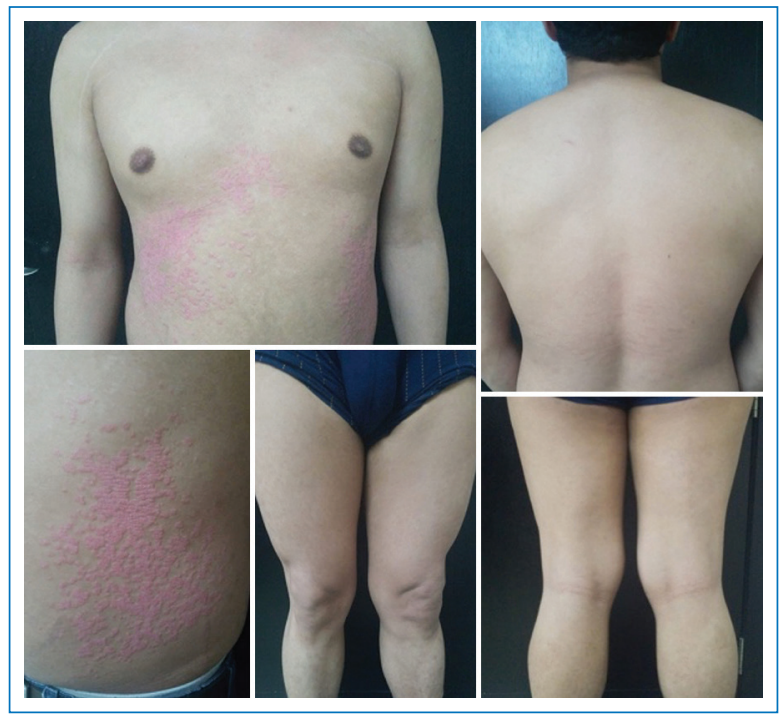

Figura 3. Remisión clínica luego de cuatro semanas de tratamiento con etanercept. Aclaramiento progresivo de las lesiones y persistencia de algunas placas eritematodescamativas en abdomen.

afectación puede ser tan extensa que se convierte en una eritrodermia, con áreas o islotes de piel sana. La hiperqueratosis de las palmas de las manos y las plantas de los pies es una característica notoria, aunque puede estar ausente, y puede relacionarse con escamas en el cuero cabelludo, además de prurito, sensación urente, engrosamiento de la lámina ungueal y hemorragias en astilla ${ }^{3,5}$. La infección aguda o el traumatismo pueden iniciar o precipitar la afección. Algunas veces, la curación parcial de las lesiones puede tener como resultado la transformación de la forma juvenil típica (tipo III) en la forma juvenil circunscrita (tipo IV) ${ }^{6}$.

El diagnóstico es clínico y se complementa con los hallazgos histopatológicos típicos, los cuales muestran acantosis e hipergranulosis confluente o focal, alternancia entre ortoqueratosis y paraqueratosis en dirección horizontal y vertical, folículos pilosos dilatados, tapones queratósicos, infiltrado de linfocitos y macrófagos perivasculares y perifoliculares ${ }^{3}$.

No existe en la actualidad un tratamiento aceptado general debido a la baja incidencia de la enfermedad, casos subdiagnosticados y curso variable de la enfermedad. Están disponibles diferentes tratamientos que muestran resultados variables. Por lo regular, en las formas leves o localizadas, se aplica tratamiento tópico: corticoides, queratolíticos, emolientes, retinoides, calcipotriol, entre otros. La fototerapia es otra de las opciones terapéuticas. Los retinoides sistémicos han demostrado en ciertos casos resultados favorables ${ }^{5,7}$. Se ha 
informado que el tratamiento con inmunosupresores (metotrexato o ciclosporina) es moderadamente efectivo en la PRP; sin embargo, el tratamiento con fármacos biológicos es más eficaz, sobre todo en pacientes con fracaso terapéutico ${ }^{8}$. Una revisión reciente recomienda un algoritmo de tratamiento que comienza con retinoides (sobre todo isotretinoína) como tratamiento de primera línea, tratamiento inmunosupresor (p. ej., metotrexato) como segunda línea y tratamiento con productos biológicos (ustekinumab, inhibidores de TNF- $\alpha$, secukinumab) como tercera línea ${ }^{9}$. Ante el fracaso terapéutico de primera y segunda líneas, puede considerarse la administración de compuestos biológicos. En una revisión sistemática se advirtió que el tratamiento con estos últimos tiene una eficacia comparable a la de la isotretinoína; sin embargo, no debe perderse de vista que las características de los pacientes difieren entre los grupos, ya que los pacientes que reciben productos biológicos se han sometido antes a más tratamientos sistémicos. Por lo tanto, los compuestos biológicos podrían ser útiles como tratamiento de tercera línea para la PRP, tras considerar la experiencia limitada y el aumento de los costos $^{10}$. En la actualidad, los inhibidores del factor de necrosis tumoral constituyen fármacos terapéuticos efectivos para casos graves resistentes a las modalidades habituales, con excelentes resultados ${ }^{11-13}$.

Debido a la escasa frecuencia de casos, no se han realizado estudios clínicos aleatorios y su tratamiento actual es desafiante y sobre todo empírico. El tratamiento de la PRP con etanercept debe aún dilucidarse. Sin embargo, el caso presentado revela que el tratamiento anti-TNF puede ser eficaz y seguro en pacientes jóvenes con PRP de tipo III en los que los medicamentos de primera y segunda líneas son ineficaces o están contraindicados.

\section{Conclusiones}

Este caso corresponde a una PRP juvenil de tipo III de presentación infrecuente en un adolescente, con manifestaciones clínicas características e histopatología consistente, y también antecedente de fracaso con retinoides sistémicos y fármacos tópicos. Se decidió iniciar ciclosporina sin mejoría durante cuatro semanas, por lo que se inició tratamiento con etanercept (50 mg/sem); luego de cuatro semanas del inicio del anti-TNF- $\alpha$ se observó mejoría clínica con aclaramiento progresivo de las lesiones. Hasta el momento, el paciente ha evolucionado de modo favorable, con buen control de enfermedad al noveno mes de seguimiento y sin efectos colaterales; en consecuencia, se considera que el etanercept es una alternativa terapéutica que puede ser eficaz y seguro en jóvenes con PRP de tipo III con fracaso a compuestos terapéuticos de primera y segunda líneas; no obstante, el papel del etanercept en la PRP todavía debe precisarse.

\section{Financiamiento}

Los autores declaran que el estudio lo financiaron ellos mismos.

\section{Conflicto de intereses}

Los autores declaran no tener conflicto de intereses.

\section{Responsabilidades éticas}

Protección de personas y animales. Los autores declaran que para esta investigación no se han realizado experimentos en seres humanos ni en animales.

Confidencialidad de los datos. Los autores declaran que han seguido los protocolos de su centro de trabajo sobre la publicación de datos de pacientes.

Derecho a la privacidad y consentimiento informado. Los autores han obtenido el consentimiento informado de los pacientes o sujetos referidos en el artículo. Este documento obra en poder del autor de correspondencia.

\section{Bibliografía}

1. Griffiths W. Pityriasis rubra pilaris. Clin Exp Dermatol. 1980:5:105-12.

2. Misery L, Faure M, Claudy A. Pityriasis rubra pilaris and human immunodeficiency virus infection - type 6 pityriasis rubra pilaris? Br J Dermatol. 1996;135(6):1008-9.

3. Sehgal VN, Srivastava G. (Juvenile) pityriasis rubra pilaris. Int J Dermatol. 2006;45(4):438-46.

4. Pajares F, Cardoza P. Pitiriasis rubra pilaris típica juvenil : reporte de un caso. 2012;23(2):87-92.

5. Allison DS, El-Azhary RA, Calobrisi SD, Dicken $\mathrm{CH}$. Pityriasis rubra pilaris in children. J Am Acad Dermatol. 2002;47(3):386-9.

6. Vila-Mas T, García-Muret P, Alomar-Muntañola A. Pitiriasis rubra pilar infantil. Piel. 2003;18(8):409-15.

7. Goldsmith LA, Weinrich E, Shupack J. Pityriasis rubra pilaris response to 13-cis-retinoic acid (isotretinoin). J Am Acad Dermatol. 1982;6(4):710-5.

8. Napolitano M, Abeni D, Didona B. Biologics for pityriasis rubra pilaris treatment: a review of the literature. J Am Acad Dermatol [Internet]. 2018;79(2):353359.e11. Available from: https://doi.org/10.1016/j.jaad.2018.03.036

9. Roenneberg S. Pityriasis rubra pilaris: algorithms for diagnosis and treatment. J Eur Acad Dermatol Venereol. 2018;32(6):889-98.

10. Kromer C, Sabat R, Celis D, Mössner R. Systemic therapies of pityriasis rubra pilaris: a systematic review. JDDG - J Ger Soc Dermatology. 2019;17(3):243-59.

11. Cox V, Lesesky EB, Garcia BD, O'Grady TC. Treatment of juvenile pityriasis rubra pilaris with etanercept. J Am Acad Dermatol [Internet]. 2008;59(5 SUPPL.):113-4. Available from: http://dx.doi.org/10.1016/j. jaad.2008.06.016

12. Galilea N, Conde-Taboada A, Campos L, López-Bran E. Pityriasis rubra pilaris treated with etanercept. Piel. 2014;29(4):255-6.

13. Lora V, De Felice C, Cota C, Graceffa D, Morrone A, Bonifati C. A case of juvenile pityriasis rubra pilaris type III successfully treated with etanercept. Dermatol Ther. 2018;31(1):1-3. 